\title{
Identification of potential microRNAs and their targets in promoting gefitinib resistance by integrative network analysis
}

\author{
Fushuang Zheng, Hongyan Zhang, Jibin Lu \\ Department of Thoracic Surgery, Shengjing Hospital of China Medical University, Shenyang 110004, China \\ Contributions: (I) Conception and design: F Zheng, J Lu; (II) Collection and assembly of data: F Zheng; (III) Data analysis and interpretation: F \\ Zheng; (IV) Collection and assembly of data: None; (V) Data analysis and interpretation: None; (IV) Manuscript writing: All authors; (V) Final \\ approval of manuscript: All authors. \\ Correspondence to: Jibin Lu. Department of Thoracic Surgery, Shengjing Hospital of China Medical University, No. 36 San Hao Street, Shenyang \\ 110004, China. Email: Lujb@sj-hospital.org.
}

\begin{abstract}
Background: Non-small cell lung cancer (NSCLC) accounts for about 80-85\% of lung cancers. Epidermal growth factor receptor tyrosine kinase inhibitors (EGFR-TKIs) such as gefitinib are considered the best choice for first-line treatment for the patients with NSCLC harboring EGFR-activating alterations. Nonetheless, $10-30 \%$ of patients may not obtain an objective response and may also experience rapid progression. The aim of our research, based on the integrative bioinformatics review, was to identify the possible miRNAs involved in gefitinib resistance.

Method: A gefitinib-resistant network composed of 15 miRNAs and 34 targets were constructed by using the bioinformatics analyses of three microarray datasets. Of these miRNAs, effects of miR-342-3p on gefitinib resistance were investigated on a gefitinib-resistant cell model (A549/GR and PC/GR cells).

Results: We reported that over-expression of miR-342-3p could significantly increase the resistance to gefitinib of A549/GR and PC9/GR cells and vice versa. Then, we recognized CPA4 as a target of hsa-miR$342-3 p$ by a luciferase reporter assay. The increase in hsa-miR-342-3p levels led to a significant reduction in CPA4 protein expression. However, the opposite results were observed upon miR-342-3p knockdown. Finally, we found that enforced CPA4 expression partially reversed miR-342-3p effects in A549/GR cells.

Conclusions: Collectively, these findings suggest that the upregulation of miR-342-3p contributes to gefitinib resistance by targeting CPA4, which may serve as a potential treatment option to overcome gefitinib resistance in patients with NSCLC.
\end{abstract}

Keywords: Gefitinib; non-small cell lung cancer (NSCLC); miR-342-3p; CPA4

Submitted Jun 16, 2019. Accepted for publication Oct 31, 2019.

doi: $10.21037 /$ jtd.2019.11.25

View this article at: http://dx.doi.org/10.21037/jtd.2019.11.25

\section{Introduction}

Lung cancer is one of the most frequent and fatal neoplasms. It is also the leading cause of cancer mortality in all parts of the world (1), with around $80-85 \%$ of lung cancers being non-small cell lung cancer (NSCLC). Despite being the most prevalent type of lung cancer, NSCLC remains a global challenge because of its high morbidity and mortality (2). Recently, molecular targeted therapy has dramatically arrived as a milestone clinical improvement for patients with precise genomic variations, especially for those patients with NSCLC-harboring epidermal growth factor receptor (EGFR)-sensitive mutations $(3,4)$. EGFRTKIs have been developed to resist epithelial-derived solid tumors for advanced NSCLC.

One of these EGFR-tyrosine kinase inhibitors (TKIs), gefitinib, is a selective inhibitor of epidermal growth factor and plays an essential role in controlling cell growth, apoptosis, and angiogenesis (5). It functions as a first-line single therapeutic agent for NSCLC (6). Unfortunately, 
resistance to therapeutic EGFR-TKIs has developed, thus limiting the usefulness of gefitinib in clinical treatment (7). For example, Yonesaka et al. found that over-expression of HER 3 could cause substantial resistance to EGFRTKIs by stimulating the downstream PI3K/AKT signaling cascades (8). However, apart from the findings of few studies like the one above, little else is understood concerning the mechanism underlying gefitinib resistance or other developed resistances to EGFR-TKI.

MiRNAs generally bind to the 3 '-untranslated regions (3'-UTRs) of target messenger RNAs (mRNAs) and cause either degradation of mRNA or inhibit translation of mRNA (9). The recent discovery of miRNAs in TKI resistance has revealed the role of non-coding RNA in gefitinib resistance in NSCLC. Garofalo et al. examined the importance of miR-30b, which was regulated by EGFR as well as MET receptor tyrosine kinases in NSCLC gefitinib resistance (10). Gao et al. explored the involvement of miR$138-5 \mathrm{p}$ in reversing the resistance to gefitinib in NSCLC (9). Recently, microarrays have been used to evaluate gene expression, demonstrating promising clinical application in cancer diagnosis and the predictive response of targeted drugs to tumor cells. They represent an innovative research approach to studying the molecular processes of therapeutic resistance in tumors (11-13).

The objective of our study was to identify likely miRNAs and their targets to promote the resistance to gefitinib in NSCLC. First, we obtained and integrated the Gene Expression Omnibus (GEO) datasets and conducted scientific bioinformatics analysis to build a gefitinibresistance miRNA-target regulatory network. Then, functional enrichment was employed to identify the GO terms and pathways of this network. The hsa-miR-342$3 p$ and its target CPA4 were selected. Finally, we found that enforced CPA4 expression partially reversed miR-342$3 p$ effects in A549/GR cells. Thus, this study reveals the impact of hsa-miR-342-3p in gefitinib-resistant NSCLC and implicates hsa-miR-342-3p as an impending treatment option for improving the effectiveness of gefitinib in NSCLC patients.

\section{Methods}

\section{Microarray data}

NCBI-GEO is a free database for next-generation sequencing. In this study, to construct a gefitinib resistancerelated network, we searched miRNA and mRNA datasets for gefitinib resistance in the GEO database (https://www. ncbi.nlm.nih.gov/geo). To ensure that the same samples were used in miRNA and mRNA datasets, three datasets, GSE74253, GSE117610, and GSE110815-all focusing on the PC9 cells-were finally selected. The sequencing data of GSE74253 and GSE117610 were based on the GPL11154 platform [Illumina HiSeq 2000 (Homo sapiens)] (11,12). The GSE74253 dataset was designed to compare the whole genome transcriptome of the gefitinib-resistant NSCLC cell line (PC9R) with its gefitinib-sensitive counterpart (PC9). The GSE117610 dataset was mainly used so that the NSCLC cell line PC9 could be made tolerant to gefitinib over 6 days. Finally, the GSE110815 dataset investigated the genome-wide miRNA expression analysis, which was performed in gefitinib-resistant sub-cell lines and gefitinibsensitive parental cell lines, based on the GPL18402 platform [Agilent-046064 Unrestricted_Human_miRNA_ V19.0_Microarray (miRNA ID version)] (13).

\section{Identification of differentially expressed genes (DEGs)}

The raw microarray data files of high throughput functional genomics expression were integrated for the analysis. The TXT format data were processed in the algorithm, and DEGs were identified. For the GSE74253 dataset, statistically significant DEGs were defined with a GFOLD value of above 1 and $6 \%$ of total detected genes. Additionally, a GFOLD value less than -1 and $5 \%$ of the total detected genes was used as a cut-off criterion. For the GSE117610 dataset, statistically significant DEGs were defined with $\mathrm{P}$ values $<0.05$, and $|\log 2 \mathrm{FC}|>1$ was set as the statistically significant threshold.

\section{Functional and patbway enrichment analyses}

Gene Ontology analysis (GO), an extremely valuable technique, is generally used for interpreting genes and gene products. It is also used to identify specific biological attributes for high-throughput genome or transcriptome data. Kyoto Encyclopedia of Genes and Genomes (KEGG) is a resource base for the methodical examination of gene functions, and it links genomic evidence with higher-order functional evidence. To investigate function annotation and pathway enrichment of the DEGs, the Database for Annotation, Visualization and Integrated Discovery (DAVID) was used to analyze the GO and KEGG enrichment analyses. Statistical significance was achieved when $\mathrm{P}<0.01$. 


\section{The miRNA-target gene interactions}

Seven databases including Targetscan (14), RNAhybrid (15), Rna22 (16), PicTar5 (17), mirBase (18), Miranda (19), and DIANA_microRNA (20) were searched to collect the miRNAtarget gene interactions. Once the appropriate processing was completed to avoid any redundancy, 289,469 miRNA-target interactions amongst 15,185 genes and 1,089 miRNAs were acquired.

\section{Cell culture}

Human non-small cell adenocarcinoma cell line A549 and PC9 were purchased from the American Type Culture Collection (Manassas, VA, USA). The culture medium was treated with different doses of gefitinib to establish gefitinib-resistant A549/GR and PC9/GR cells. The range of dosages of gefitinib ranged from 0 to $80 \mathrm{nM}$, and the dosage gradient was $25-50 \%$ of the prior dose. The subsequent bout was provided up until the cells became steady in proliferation without any substantial death $(5,21)$.

All cells were cultured by using either RPMI 1640 medium or DMEM (Life Technologies, Carlsbad, CA, USA). The medium contained $10 \%(\mathrm{v} / \mathrm{v})$ fetal bovine serum (FBS; Gibco, Grand Island, NY, USA). Cells were incubated in an incubator with humidified air of $5 \%(\mathrm{v} / \mathrm{v}) \mathrm{CO}_{2}$ at $37^{\circ} \mathrm{C}$.

\section{Plasmid construction}

As mentioned in previous studies, both wild-type and mutated CPA4 3'-UTRs were cloned into a dual-luciferase reporter vector (Promega, Madison, WI, USA) (22). Briefly, the 3'UTR of the human CPA4 gene was acquired by performing genomic PCR. The primers used were as follows: 5'-CGG AATTCATCCAGATGGCAAATGACCA-3' (forward) and 5'-CCGCTCGAGTTAGCCCCCGTGACCTCTT-3' (reverse). Moreover, these two primers, along with the other two following primers, were used to conduct recombinant PCR to introduce alterations into the seed sequences of the projected miR-342-3p target sites within CPA4 3'-UTR: 5'-TTATTTTATGAAAGTGGGCACTTGTCAAAGTG ATG-3' (forward) and 5'-CATCACTTTGACAAGTGCC CACTTTCATAAAATAA-3' (reverse). The miRNA-342-3p mimics or the antisense of miR-342-3p-5p (anti-miR-342-3p) and their analogous miRNA controls were constructed from RuiBo (Guangzhou, China). Scrambled and nontargeted irrelevant RNA sequences were used as negative controls. Anti- CPA4 (1:1,000; Cat. ab85206, ABCOM, Boston, MA,
USA) and anti-GAPDH (sc-25778) antibodies were bought from Santa Cruz Biotechnology (Dallas, TX, USA). Gefitinib was bought from Sigma-Aldrich (St. Louis, MO, USA).

\section{Luciferase reporter assay}

A549 and PC9 cells were cultured in 24-well plates at a density of $1 \times 10^{3}$ cells/well. Next, the cells were cotransfected with luciferase reporters. Co-transfection was performed either with wild-type (WT) CPA4 (CPA4WT), mutant CPA4 (CPA4-Mut), a combination with miR342-3p mimic, or a negative control (NC) group using Lipofectamine 3000. Cells were collected, and a luciferase assay kit was used to measure luciferase activity (Promega, Madison, WI, USA) as per the supplier's protocol after $48 \mathrm{~h}$.

\section{Reverse transcription-quantitative polymerase chain reaction ( $R T-q P C R)$}

Total RNA was isolated from A549 and PC9 cells by using Trizol reagent (Thermo Fisher Scientific, USA). Either the TaqMan MicroRNA Reverse Transcription Kit or TaqMan High-Capacity cDNA Reverse Transcription Kit (Thermo Fisher Scientific) was used to perform qRT-PCR. Power SYBR Green PCR master mix (Thermo Fisher Scientific, Inc.) was used to conduct qPCR for 35 cycles at $95^{\circ} \mathrm{C}$ for $30 \mathrm{sec}, 60{ }^{\circ} \mathrm{C}$ for $30 \mathrm{sec}$, and $72{ }^{\circ} \mathrm{C}$ for $35 \mathrm{sec}$. Gene expression levels were standardized with $\beta$-actin and evaluated using the $2^{-\Delta \Delta \mathrm{Cq}}$ method.

\section{CCK-8 cell viability assay}

A549 and PC9 cells were cultured into 96-well plates with a density of $2 \times 10^{4}$ cells/well. After $24 \mathrm{~h}$, cells transfected with either miR-342-3p mimic or miR-342-3p inhibitor were treated with different concentrations of gefitinib $(0,2.5,5$, $10,20,40$, and $80 \mathrm{nM})$ for another 24 hours. Cell counting kit-8 (CCK-8) (Dojindo, Kumamoto, Japan) was used to evaluate cell viability after 48 -h treatment.

\section{Acridine orange/ethidium bromide (AO/EB) staining}

miR-342-3p mimic and miR-342-3p + CPA4 or control cells $\left(1 \times 10^{5}\right.$ cells $)$ were seeded in 6 -cm culture plates and treated with gefitinib $(60 \mathrm{nM})$ for $24 \mathrm{~h}$. The next day, the cells were treated for 5 mins with $\mathrm{AO}$ and $\mathrm{EB}$ mixing solution (Solarbio of Biotechnology, Shang Hai, China). Fluorescence microscopy was used to visualize morphological changes in 
A

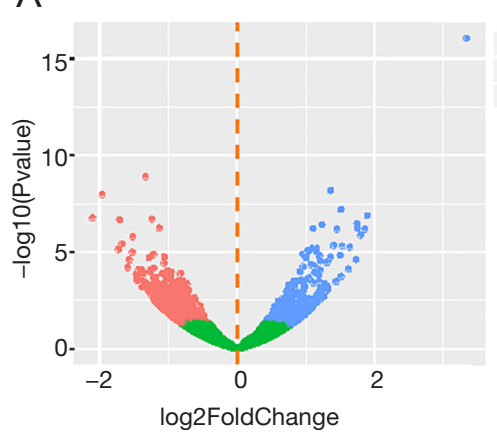



B

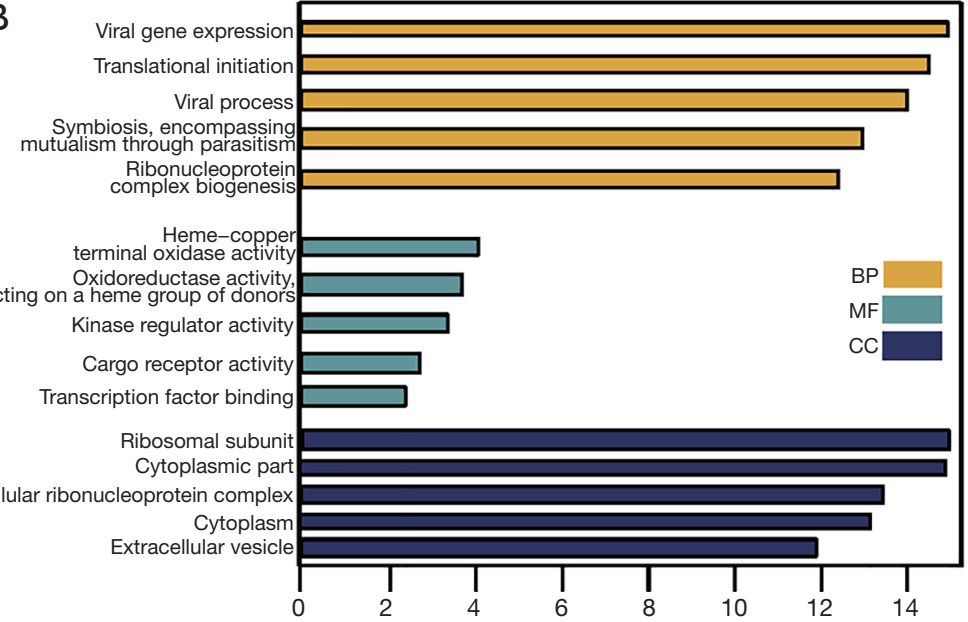

\begin{tabular}{|l|c|}
\hline DEGs & Number \\
\hline Upregulated & 595 \\
\hline Downregulated & 1095 \\
\hline
\end{tabular}

D

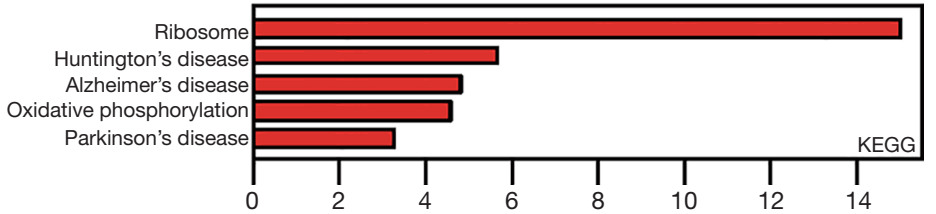

Figure 1 Identification and functional characterization of DEGs from the GSE117610 dataset. (A) Volcano plot of DEGs between gefitinibresistant NSCLC cell lines and the parental cell line. (B) The distribution of significant DEGs in gefitinib-resistant cells. (C) Top 5 GO terms and (D) KEGG enriched pathways of significant DEGs. DEG, differentially expressed gene; NSCLC, non-small cell lung cancer; GO, Gene Ontology; KEGG, Kyoto Encyclopedia of Genes and Genome.

cells at 200x magnification. Apoptotic cell percentage was determined with the following formula:

Apoptotic rate $(\%)=$ number of apoptotic cells/number of all cells counted $\times 100 \%$.

\section{Statistical data analysis}

Data were obtained from 3 to 6 independent experiments. Results are represented as the mean \pm standard deviation. Data were analyzed by unpaired Student's $t$-test. Statistical comparisons among multiple groups were performed by analysis of variance (ANOVA) followed by Tukey's multiple comparison test. Spearman's rank correlation tests were used to analyze miR-342-3p and the IC50 of gefitinib. Statistical significance was achieved when $\mathrm{P}<0.05$.

\section{Results}

\section{Identification and functional characterization of upregulated DEGs in gefitinib-resistant NSCLC}

In order to identify the candidate genes that confer gefitinib resistance in NSCLC, the GSE117610 dataset containing the gefitinib-resistant cells and their parental cells was obtained. Then, 1,690 DEGs were identified based on 2-fold change (Figure 1A). Out of these 1,690 DEGs, 595 were upregulated, and 1,095 were downregulated (Figure $1 B$ ). To get insights into the functions of these significant DEGs, we uploaded all DEGs on DAVID to recognize the overrepresented GO categories and KEGG pathways. This process identified the top 5 GO terms and enrichment pathways, and also indicated that DEGs were highly related to viral gene expression, translational initiation, and ribosomal subunits (Figure 1C). In addition, as shown in Figure 1D, the significant DEGs were also augmented in ribosome and oxidative phosphorylation (Figure 1D).

For the GSE74253 dataset, there were 1,487 significantly upregulated and 1,112 significantly downregulated genes (Figure 2A). After comparing the significant DEGs in GSE117610, the intersecting genes were obtained (Figure 2B). GO and KEGG results also indicated the significant DEGs of GSE74253 dataset were highly related to cell-cell adhesion and ECM-receptor interaction, along 
A

\begin{tabular}{|l|c|}
\hline DEGs & Number \\
\hline Upregulated & 1487 \\
\hline Downregulated & 1112 \\
\hline
\end{tabular}

C

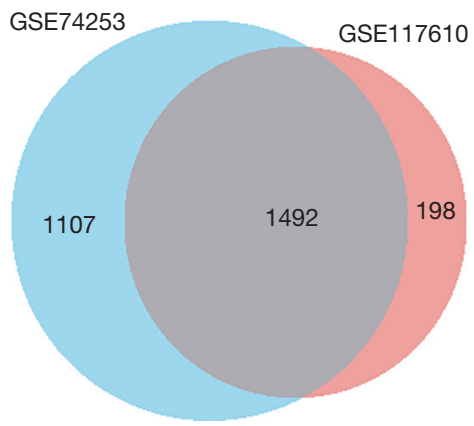

B

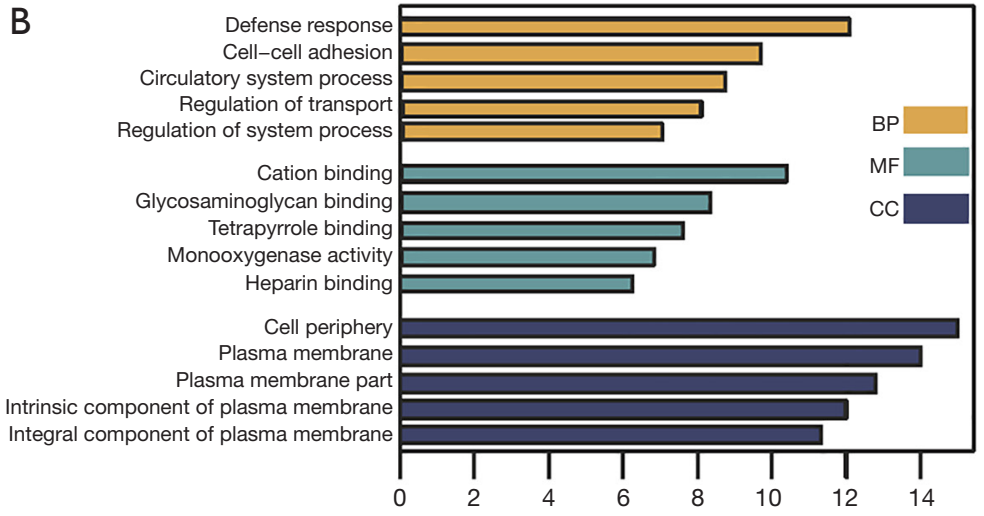

D

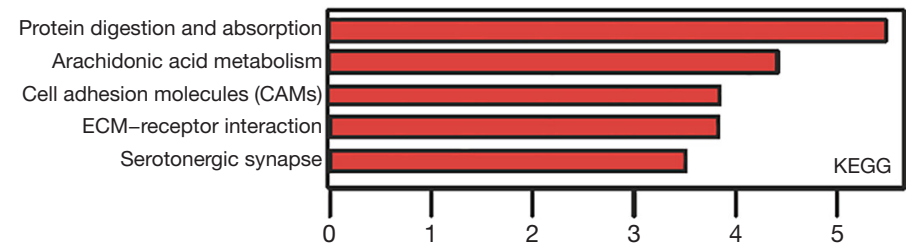

Figure 2 Identification and functional characterization of DEGs from the GSE74253 dataset. (A) The distribution of significant DEGs in gefitinib-resistant cells. (B) Venn diagram of significant DEGs between GSE74253 and GSE117610. (C) Top 5 GO terms and (D) KEGG enriched pathways of significant DEGs. DEG, differentially expressed gene; GO, Gene Ontology; KEGG, Kyoto Encyclopedia of Genes and Genome.

with cell adhesion molecules (CAMs) (Figure 2C,D).

\section{Construction of the miRNA-target regulatory network}

To construct the miRNA-target differential regulatory network, differentially expressed miRNAs were first identified. From the GSE110815 dataset, 32 significantly upregulated and 44 downregulated miRNAs were recognized (Figure $3 A, B$ ).

We then constructed all the terms of miRNA-target interactions included across the 7 databases. Only the miRNA-target associations recorded in more than 2 databases were retained. Then, we mapped the significant differentially expressed miRNAs and intersecting DEGs into the above miRNA-target interactions. As a result, 43 unique miRNA-target associations were finally obtained from 289469 primary terms. The 43 miRNA-target associations were composed of 15 miRNAs and 34 targets (Figure 3C). Finally, for the miRNA-target regulation network, the degrees of all miRNAs ranged from 1 to 5 , while the degrees of all target genes ranged from 1 to 2 .
This suggests that miRNAs as regulators tend to deregulate more, and some miRNAs may act as important regulators for multiple target genes, including hsa-miR-128's 5 regulation genes (POU2F3, HMGB3, FNDC4, YPEL3, NAV3). Meanwhile, some miRNAs may act as specific regulators to single genes, whereas some target genes may be regulated by multiple miRNAs. GO, and KEGG results showed these targets in the network were enriched into intracellular signal transduction and pathway of PPAR signaling along with proteoglycans in cancer (Figure 3D).

\section{miR-342-3p enhances gefitinib resistance in A549 and PC9 cells}

To examine the involvement of miR-342-3p in gefitinib resistance in NSCLC A549 and PC9 cells, we first established an NSCLC gefitinib resistance cell line (A549/ GR and PC9/GR) $(5,21)$ (Figure S1). We evaluated the effect of miR-342-3p in A549/GR and PC9/GR cells. Our data showed that increased expression of miR-342-3p significantly augmented the resistance to gefitinib of A549/ 

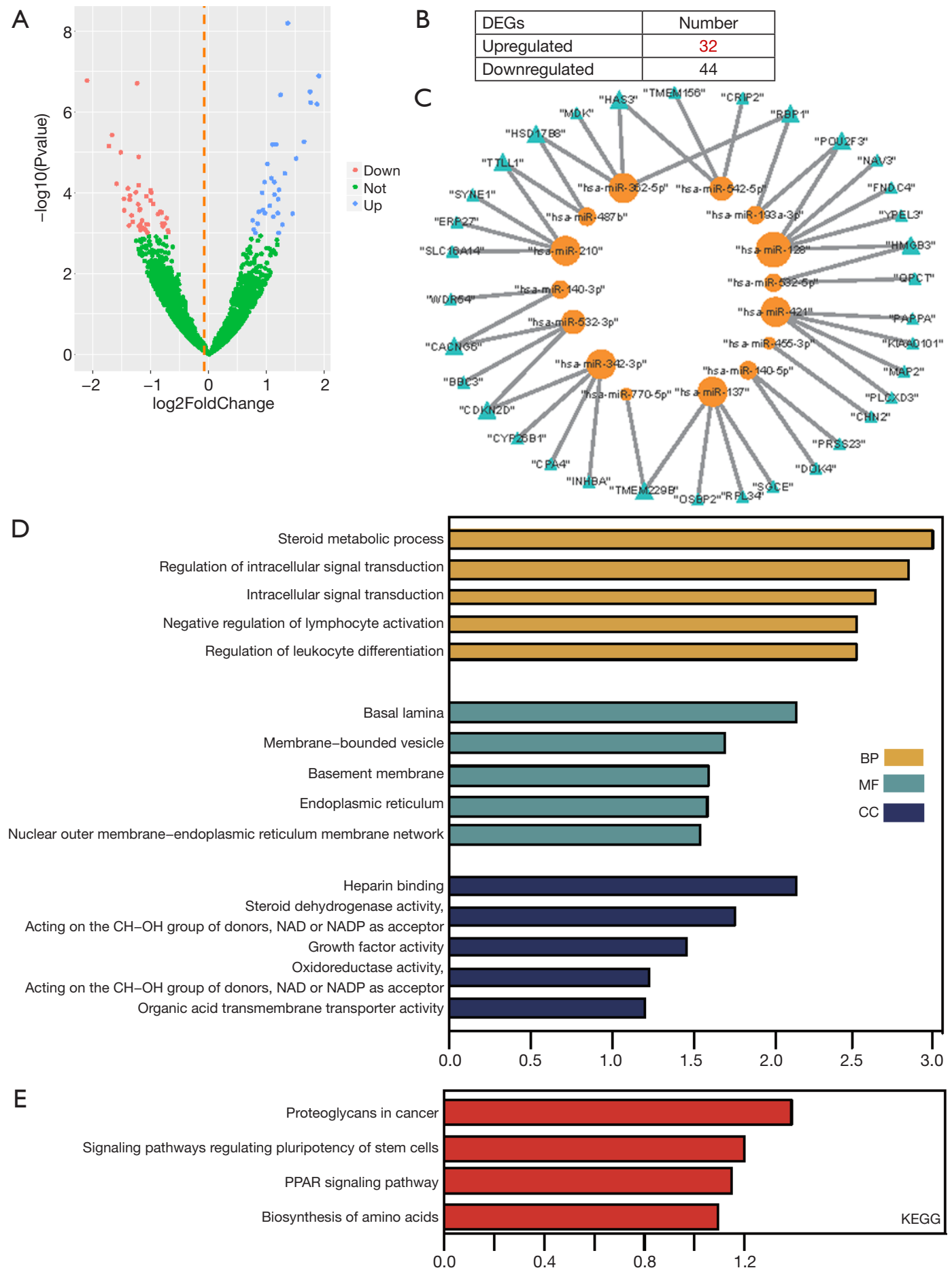

Figure 3 Construction and functional characterization of the gefitinib-resistant miRNA-target network. (A) Volcano plot of DEGs between gefitinib-resistant NSCLC cell lines and the parental cell line. (B) The distribution of significant miRNAs in gefitinib-resistant cells. (C) Gefitinib-resistant miRNA-target network. Yellow nodes represent miRNAs, while blue nodes represent mRNA targets. (D) Top 5 GO terms and (E) KEGG enriched pathways of mRNAs in the network. DEG, differentially expressed gene; NSCLC, non-small cell lung cancer; GO, Gene Ontology; KEGG, Kyoto Encyclopedia of Genes and Genome. 



Figure 4 miR-342-3p enhances gefitinib resistance in A549 and PC9 cells. (A and B) miR-342a-3p-overexpressing A549/GR (A) or PC9/GR (B) lung cancer cells were treated with increasing concentrations of gefitinib. Cell viability assays were performed by the CCK-8 kit. (C and D) Cell viability assay of A549/GR (C) or PC9/GR (D) cells transfected with miR-342-3p and treated and analyzed as in (A). Experiments were repeated three times. $\mathrm{n}=3-6,{ }^{*} \mathrm{P}<0.05$ versus the $\mathrm{NC}$ group; **, $\mathrm{P}<0.01$. CCK-8, cell counting kit-8; NC, negative control.

GR and PC9/GR cells (Figure 4A,B). However, decreased expression of the miR-342-3p inhibited the viability of the A549/GR and PC9/GR cells that were treated with various dosages of gefitinib for $24 \mathrm{~h}$ compared to the NC group (Figure 4C,D). These data reveal that miR-342-3p might be an agent in gefitinib resistance in lung cancer cells.

\section{Identification of CPA4 as a target of bsa-miR-342-3p}

To further confirm the association between CPA4 and miR$342-3 p$, we first inspected the manifestation of miR-342$3 \mathrm{p}$ after transfection with miR-342-3p mimic or inhibitor (Figure $5 A$ ). We then measured the mRNA level of CPA4 after miR-342-3p knockdown or overexpression in A549 and PC9 cells (Figure 5B,C). When compared to the NC group, the mRNA, along with the protein level of CPA4, was significantly up-regulated in the miR-342-3p inhibitor group and significantly down-regulated in the miR-342$3 \mathrm{p}$ mimic group $(\mathrm{P}<0.01)$. These data suggest that $\mathrm{CPA} 4$ is regulated by miR-342-3p (Figure $5 D, E$ ).

The bioinformatics analysis results showed that miR$342-3 p$ contained the specific binding sequence of the 3 'UTR regions of the CPA4 gene (Figure $5 F$ ). A luciferase reporter assay showed that the expression of miR-342-3p diminished the luciferase activity of the 3'-UTR of CPA4. Nevertheless, the miR-342-3p-Mut did not inhibit the manifestation of $\mathrm{CP} 4$ on luciferase activity (Figure $5 G, H$ ).

\section{Enforced CPA4 expression partially reversed miR-342-3p effects in A549/GR cells}

To investigate if the involvement of miR-342-3p in A549/GR cells was through CPA4, a CPA4 expression vector was co-transfected with miR-342-3p. First, we found that the viability of A549/GR cells was reversed by AO/EB (Figure $6 A, B$ ). Meanwhile, we confirmed that the expression of miR-342-3p expression was restored by CPA4 expression vector co-transfection after treatment with or without gefitinib (Figure 6C).

\section{Discussion}

Even though many effective types of medication are available to treat tumors via the targeting of various molecules, many patients still acquire permanent resistance towards certain treatments (22). NSCLC prognosis remains unfavorable since it is a systemic disease rendered uncontrollable by such drug resistance. This study identified miR-342-3p as a prospective molecule for conferring gefitinib resistance in NSCLC.

We firstly identified 1,690 DEGs from the GSE117610 dataset, and GO and KEGG pathway enrichment were implemented. Of these DEGS, those that were significant were found to be highly related to GO BP terms like viral gene expression, translational initiation, 




Figure 5 Identification of CPA4 as a target of hsa-miR-342-3p. (A) The expression of miR-342-3p after transfecting with miR-342 mimic or inhibitor by qRT-PCR. (B,C) The expression of CPA4 after transfecting with miR-342 mimic or inhibitor by qRT-PCR in A549 and PC9 cells. (D,E) The protein level of CPA4 after transfecting with miR-342 mimic or inhibitor by western blot in A549 and PC9 cells. (F) The seed unit between miR-342-3p and CPA4. (G,H) A schematic diagram of the CPA4 3'-UTR luciferase reporter constructs are shown in A549 and PC9 cells. Experiments were repeated three times. $n=3-6,{ }^{*} \mathrm{P}<0.05$ versus NC group or control group. NC, negative control; UTR, untranslated region.

and ribosomal subunits (Figure 1C), along with pathways including ribosome and oxidative phosphorylation (Figure 1D). Animals use ATP generated from oxidative phosphorylation to maintain day-to-day activities; thus, it is accountable for preserving metabolic homeostasis (23). Abnormal metabolism is a hallmark of cancer, and aberrant phosphorylation in A549/GR was found to be related to the ineffectiveness of gefitinib (24-26). For the GSE74253 dataset, there were 1,487 significantly upregulated and 1,112 significantly downregulated genes (Figure $2 A$ ). The results obtained from GO and KEGG indicated that the significant DEGs were highly related to cell-cell adhesion, ECM-receptor interaction, and CAMs (Figure 2B,C). ECMreceptor interaction pathway was identified in crizotinib- resistant NSCLC cells and trastuzumab-resistant gastric cancer cells $(27,28)$. Several cell-adhesion molecules are also recognized to be effective clinical biomarkers and are thus promising treatment options for lung, colorectal, and pancreatic cancer (29).

To construct the miRNA-target differential regulatory network, the differentially expressed miRNAs were first identified. From the dataset GSE110815, 32 significantly upregulated and 44 downregulated miRNAs were found (Figure $3 A, B$ ). We then integrated the 7 databases to obtain miRNA-target interactions. Subsequently, 43 unique miRNA-target associations, composed of 15 miRNAs and 34 targets, were obtained from 289,469 primary terms (Figure 3C). Finally, for the miRNA-target regulation 

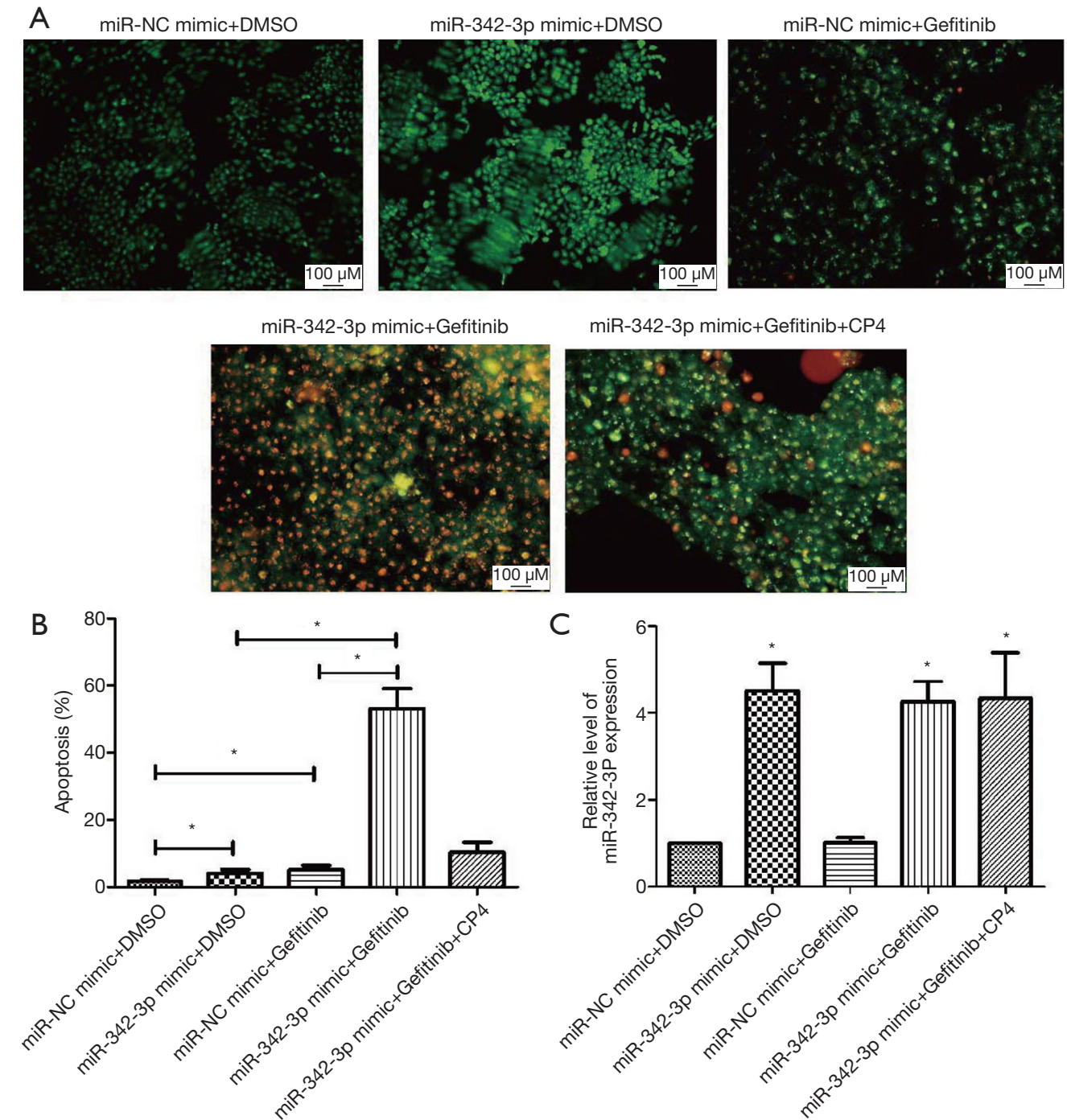

miR-342-3p mimic+Gefitinib+CP4


Figure 6 Enforced CPA4 expression partially reversed miR-342-3p effects in A549/GR cells. (A,B) The apoptosis cell of A549/GR after treating with different groups by AO/EB. (C) The expression of miR-342-3p. ${ }^{*} \mathrm{P}<0.05$ versus the NC group or control group. AO/EB, acridine orange/ethidium bromide; NC, negative control.

network, the degrees of all miRNA ranged from 1 to 5 , of which hsa-miR-128 regulated 5 genes (POU2F3, HMGB3, FNDC4, YPEL3, NAV3). Chen et al. reported that miR-128a confers chemoradiotherapy sensitivity of laryngeal cancer cells, and targeting the BMI1 gene is a possible mechanism of the effect (30). Furthermore, Jiang et al. reported that compared to the non-CSCs, there was substantial inhibition miR-128 in the PC9-CSCs and suggested that the miR-128/c-met pathway augments the sensitivity of gefitinib towards the lung cancer stem cells through inhibiting the PI3K/AKT pathway (31). There were 5 miRNAs with a degree of 4 . Of these miRNAs, miR-
$342-3 p$ has been found to be involved in the pathogenesis and drug resistance of NSCLC. Han et al. reported that miR-342-3p targeted ATF3, which promotes osteogenic differentiation (32). In addition, colleagues from Zhang et al.'s labs also found that miR-342-3p, as a ceRNA agent in IncRNA SCARNA2, intermediates colorectal cancer chemoresistance (33). In a previous study, LINC00460 regulated KDM2A to stimulate cell proliferation and migration by pursuing miR-342-3p in gastric cancer (34). However, the involvement of miR-342-3p in gefitinib resistance for NSCLC is not well understood.

miR-342-3p has been found to be involved in the 
pathogenesis and drug resistance of NSCLC. We reported that the significant DEGs were enriched in the intracellular signal transduction and pathway of PPAR signaling, along with the proteoglycans in cancer (Figure 3D). Peroxisome proliferator-activated receptor gamma (PPAR $\gamma)$ is known to regulate the metabolism of glucose and lipid, inflammation, in addition to endothelial function (35). Efatutazone and gefitinib are agonists of PPAR $\gamma$, and both together could prevent the propagation of the cells which are resistant to EGFR-TKI through the PPAR $\gamma /$ PTEN/Akt pathway (36). These results indicate that our miRNA-target network provides insights into the miRNA-related mechanisms underlying gefitinib resistance in NSCLC. Here, we are first to reveal that the overexpression of miR-342-3p can significantly increase the gefitinib resistance of A549/GR and PC9/GR cells (Figure 4).

We then selected CPA4, a member of the carboxypeptidase A family (37), as a potential target of miR-342-3p. Because the upregulation of the expression of CPA4 in NSCLC is related to a negative prediction, serum CPA4 level along with serum CYFRA21-1 level may be additionally used for the timely discovery of NSCLC (38). In our data, we found that CPA4 was regulated by miR342-3p (Figure 5). These data reveal that CPA4 is a straight target gene of miR-342-3p. In previous years, the prevention of cell-death stimulation by gefitinib was the main concern of cancer drug resistance research (39). We also observed the cell apoptosis of NSCLC after the upregulation of CPA4 in gefitinib-resistant lung cancer cells compared to the treatment with miR-342-3p knockdown or up-regulation (Figure 6).

In conclusion, we identified gefitinib resistance-related miRNA and genes and then constructed a miRNAtarget network. We demonstrated that miR-342-3p may confer gefitinib resistance in NSCLC via targeting CPA4. Nevertheless, additional studies, such as in vivo experiments aimed to explain the mechanism of drug resistance in up/ down streams of miR-342-3p in gefitinib-resistant NSCLC animal models, are still needed.

\section{Acknowledgments}

None.

\section{Footnote}

Conflicts of Interest: The authors have no conflicts of interest to declare.
Ethical Statement: The authors are accountable for all aspects of the work in ensuring that questions related to the accuracy or integrity of any part of the work are appropriately investigated and resolved.

\section{References}

1. Agustoni F, Suda K, Yu H, et al. EGFR-directed monoclonal antibodies in combination with chemotherapy for treatment of non-small-cell lung cancer: an updated review of clinical trials and new perspectives in biomarkers analysis. Cancer Treat Rev 2019;72:15-27.

2. Torre LA, Bray F, Siegel RL, et al. Global cancer statistics, 2012. CA Cancer J Clin 2015;65:87-108.

3. Zito Marino F, Ronchi A, Accardo M, et al. Concomitant ALK/KRAS and ALK/EGFR mutations in non small cell lung cancer: different profile of response to target therapies. Transl Cancer Res 2017;6:S457-60.

4. Yang RF, Yu B, Zhang RQ, et al. Bevacizumab and gefitinib enhanced whole-brain radiation therapy for brain metastases due to non-small-cell lung cancer. Braz J Med Biol Res 2017;51:e6073.

5. Ma G, Zhu J, Liu F, et al. Long Noncoding RNA LINC00460 Promotes the Gefitinib Resistance of Nonsmall Cell Lung Cancer Through Epidermal Growth Factor Receptor by Sponging miR-769-5p. DNA Cell Biol 2019;38:176-83.

6. Peng K, Tian X, Qian Y, et al. Novel EGFR inhibitors attenuate cardiac hypertrophy induced by angiotensin II. J Cell Mol Med 2016;20:482-94.

7. Lu H, Liu S, Zhang G, et al. PAK signalling drives acquired drug resistance to MAPK inhibitors in BRAFmutant melanomas. Nature 2017;550:133-6.

8. Yonesaka K, Hirotani K, Kawakami H, et al. AntiHER3 monoclonal antibody patritumab sensitizes refractory non-small cell lung cancer to the epidermal growth factor receptor inhibitor erlotinib. Oncogene 2016;35:878-86.

9. Gao Y, Fan X, Li W, et al. miR-138-5p reverses gefitinib resistance in non-small cell lung cancer cells via negatively regulating $\mathrm{G}$ protein-coupled receptor 124 . Biochem Biophys Res Commun 2014;446:179-86.

10. Garofalo M, Romano G, Di Leva G, et al. EGFR and MET receptor tyrosine kinase-altered microRNA expression induces tumorigenesis and gefitinib resistance in lung cancers. Nat Med 2011;18:74-82.

11. Song KA, Hosono Y, Turner C, et al. Increased Synthesis of MCL-1 Protein Underlies Initial Survival of EGFR- 
Mutant Lung Cancer to EGFR Inhibitors and Provides a Novel Drug Target. Clin Cancer Res 2018;24:5658-72.

12. Qi M, Tian Y, Li W, et al. ERK inhibition represses gefitinib resistance in non-small cell lung cancer cells. Oncotarget 2018;9:12020-34.

13. Zhi H, Li X, Wang P, et al. Lnc2Meth: a manually curated database of regulatory relationships between long noncoding RNAs and DNA methylation associated with human disease. Nucleic Acids Res 2018;46:D133-8.

14. Lewis BP, Shih IH, Jones-Rhoades MW, et al. Prediction of mammalian microRNA targets. Cell 2003;115:787-98.

15. Rehmsmeier M, Steffen P, Hochsmann M, et al. Fast and effective prediction of microRNA/target duplexes. RNA 2004;10:1507-17.

16. Miranda KC, Huynh T, Tay Y, et al. A pattern-based method for the identification of MicroRNA binding sites and their corresponding heteroduplexes. Cell 2006;126:1203-17.

17. Krek A, Grun D, Poy MN, et al. Combinatorial microRNA target predictions. Nat Genet 2005;37:495-500.

18. Kozomara A, Griffiths-Jones S. miRBase: integrating microRNA annotation and deep-sequencing data. Nucleic Acids Res 2011;39:D152-7.

19. Betel D, Wilson M, Gabow A, et al. The microRNA. org resource: targets and expression. Nucleic Acids Res 2008;36:D149-53.

20. Maragkakis M, Vergoulis T, Alexiou P, et al. DIANAmicroT Web server upgrade supports Fly and Worm miRNA target prediction and bibliographic miRNA to disease association. Nucleic Acids Res 2011;39:W145-8.

21. Chandimali N, Huynh DL, Zhang JJ, et al. MicroRNA-122 negatively associates with peroxiredoxinII expression in human gefitinib-resistant lung cancer stem cells. Cancer Gene Ther 2019;26:292-304.

22. Nakatani K, Yamaoka T, Ohba M, et al. KRAS and EGFR Amplifications Mediate Resistance to Rociletinib and Osimertinib in Acquired Afatinib-Resistant NSCLC Harboring Exon 19 Deletion/T790M in EGFR. Mol Cancer Ther 2019;18:112-26.

23. Wilson DF. Oxidative phosphorylation: regulation and role in cellular and tissue metabolism. J Physiol 2017;595:7023-38.

24. Giordano CR, Mueller KL, Terlecky LJ, et al. A targeted enzyme approach to sensitization of tyrosine kinase inhibitor-resistant breast cancer cells. Exp Cell Res 2012;318:2014-21.

25. Zhang L, Li J, Hu J, et al. Cigarette smoke extract induces EGFR-TKI resistance via promoting EGFR signaling pathway and ROS generation in NSCLC cell lines. Lung Cancer 2017;109:109-16.

26. Yu L, Lu M, Jia D, et al. Modeling the Genetic Regulation of Cancer Metabolism: Interplay between Glycolysis and Oxidative Phosphorylation. Cancer Res 2017;77:1564-74.

27. Yu C, Xue P, Zhang L, et al. Prediction of key genes and pathways involved in trastuzumab-resistant gastric cancer. World J Surg Oncol 2018;16:174.

28. Wei J, van der Wekken AJ, Saber A, et al. Mutations in EMT-Related Genes in ALK Positive Crizotinib Resistant Non-Small Cell Lung Cancers. Cancers (Basel) 2018. doi: 10.3390/cancers 10010010 .

29. Beauchemin N, Arabzadeh A. Carcinoembryonic antigenrelated cell adhesion molecules (CEACAMs) in cancer progression and metastasis. Cancer Metastasis Rev 2013;32:643-71.

30. Chen H, Jin L, Zhou L, et al. Overexpressed miR128a enhances chemoradiotherapy to laryngeal cancer cells and its correlation with BMI1. Future Oncol 2018;14:611-20.

31. Jiang J, Feng X, Zhou W, et al. MiR-128 reverses the gefitinib resistance of the lung cancer stem cells by inhibiting the c-met/PI3K/AKT pathway. Oncotarget 2016;7:73188-99.

32. Han Y, Zhang K, Hong Y, et al. miR-342-3p promotes osteogenic differentiation via targeting ATF3. FEBS Lett 2018;592:4051-65.

33. Zhang PF, Wu J, Wu Y, et al. The lncRNA SCARNA2 mediates colorectal cancer chemoresistance through a conserved microRNA-342-3p target sequence. J Cell Physiol 2019;234:10157-65.

34. Wang F, Liang S, Liu X, et al. LINC00460 modulates KDM2A to promote cell proliferation and migration by targeting miR-342-3p in gastric cancer. Onco Targets Ther 2018;11:6383-94.

35. Wang S, Dougherty EJ, Danner RL. PPARgamma signaling and emerging opportunities for improved therapeutics. Pharmacol Res 2016;111:76-85.

36. Ni J, Zhou LL, Ding L, et al. PPARgamma agonist efatutazone and gefitinib synergistically inhibit the proliferation of EGFR-TKI-resistant lung adenocarcinoma cells via the PPARgamma/PTEN/Akt pathway. Exp Cell Res 2017;361:246-56.

37. Tanco S, Zhang X, Morano C, et al. Characterization of the substrate specificity of human carboxypeptidase A4 and implications for a role in extracellular peptide processing. $\mathrm{J}$ Biol Chem 2010;285:18385-96. 
38. Sun L, Wang Y, Yuan H, et al. CPA4 is a Novel Diagnostic and Prognostic Marker for Human Non-Small-Cell Lung Cancer. J Cancer 2016;7:1197-204.

39. Ajabnoor GM, Crook T, Coley HM. Paclitaxel resistance

Cite this article as: Zheng F, Zhang $\mathrm{H}$, Lu J. Identification of potential microRNAs and their targets in promoting gefitinib resistance by integrative network analysis. J Thorac Dis 2019;11(12):5535-5546. doi: 10.21037/jtd.2019.11.25 is associated with switch from apoptotic to autophagic cell death in MCF-7 breast cancer cells. Cell Death Dis 2012;3:e260. 

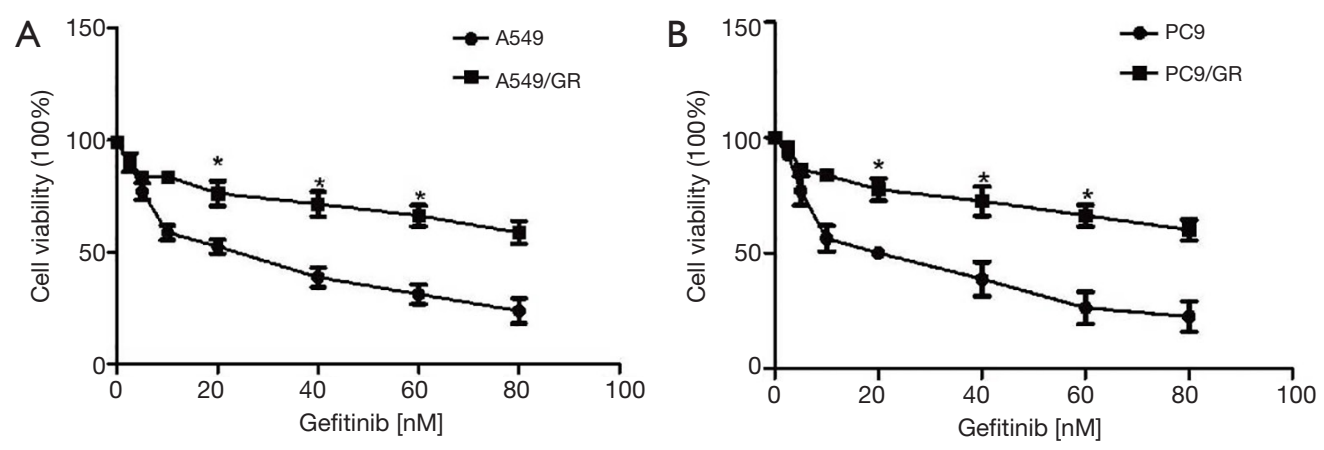

Figure S1 The establishment of A549/GR and PC9/GR. (A,B) The cell viability of A549/GR and PC9/GR after treating with gefitinib. $\mathrm{n}=3-6,{ }^{*} \mathrm{P}<0.05$ versus the A549/GR or PC9/GR group. 